Albrecht Greule

Regensburg

\title{
Valenzgeschichte, Sprachgeschichte und historische Syntax
}

DOI: 10.14232/fest.bassola. 9

\begin{abstract}
Der Beitrag greift die Feststellung von Péter Bassola auf, dass die wichtigsten Valenzinformationen die Satzbaupläne sind, und überträgt sie in die deutsche Sprachgeschichte. Dadurch kann die historische Valenzlexikographie, die zum Stillstand gekommen ist, belebt werden. An den beiden Verben zahlen und bieten wird gezeigt, wie auf der Grundlage des Deutschen Wörterbuchs online und der Sprachstadienwörterbücher online Einträge für das geplante Historisch syntaktische Verbwörterbuch und in der Folge „Valenzgeschichten“, die den Verbwandel syntaktisch und semantisch beschreiben, ausgearbeitet werden können.
\end{abstract}

\section{Historische Valenz}

Das Geburtstagsjubiläum von Péter Bassola ist ein würdiger Anlass zurückzuschauen, welchen Stand die Forschungen zur historischen Valenz erreicht haben, und nach vorne zu blicken: Welche Forschungsdesiderate bestehen noch? Ohne es beabsichtigt zu haben, weist der Jubilar in seinem Beitrag zum Handbuch „Dependenz und Valenz" der historischen Valenz-Lexikographie mit folgender Äußerung zum Verb als Valenzträger im Wörterbuch den Weg:

Die wichtigsten Valenzinformationen für Verben sind die Satzbaupläne (SBP) mit Informationen zu den obligatorischen und fakultativen (in runden Klammern) Ergänzungen und mit Angabe der Ausdrucksformen der einzelnen Argument (Bassola 2006: 1388).

Genauso wie Péter Bassola wollen wir im Folgenden einen vom VALBU: Valenzwörterbuch deutscher Verben ausgehenden Vergleich ausführen, der 
aber nicht synchron, sondern diachron - auf die Satzbaupläne abzielend angelegt ist.

Der Rückblick auf das bis heute in der historischen Valenzforschung Erreichte ist im zweiten Band des Handbuchs zur Dependenz und Valenz in der Kategorie „Das Valenzkonzept in der Sprachgeschichtsforschung“ festgehalten. Mehrere Artikel betreffen den Valenzwandel sowie Fallstudien zur Valenz in den deutschen Sprachperioden (vgl. Ágel et al. 2006: 1447-1522). In der Kategorie „Das Valenzkonzept in der Lexikographie“ vermisst man zumindest Überlegungen zu einem historischen deutschen Valenzlexikon, aus dem der Valenzwandel der Verben abzulesen wäre. Eine Richtung, wie ein solches historisches Valenzlexikon strukturiert sein könnte, nämlich nach Wortfeldern, deutet Nándor Cisky mit seinen Studien zur Struktur des Wortfelds ,wachsen (Csiky 2008, vgl. auch Greule 2014: 59-62) an. Pläne zu Wörterbüchern der einzelnen deutschen Sprachstadien, sieht man von einem Valenzwörterbuch zu den ahd. Texten des 9.Jh. (Greule 1999) ab, sind nicht verwirklicht worden. Ganz an der Valenz orientiert sich aber die Darstellung des Satzbaus in der Einführung in die deutsche Sprachgeschichte von Hans Ulrich Schmid (2017: 185-201).

Eine Ursache für das Stocken im Bereich der historischen Valenzlexikographie liegt bei der inzwischen erheblich gewachsenen Bedeutung des Computers im Rahmen der Digital Humanities. Durch die Förderung der DFG sind jetzt alle großen historischen Wörterbücher der deutschen Sprache, angefangen beim DWB, entweder ganz oder zu großen Teilen digital verfügbar. Das bedeutet, dass die historische Valenz-Forschung diese Digitalisate nutzen muss, indem sowohl die Ermittlung der Valenz bzw. der Satzbaupläne direkt am PC erfolgen kann (vgl. Greule 2018) als auch die Ergebnisse in einer Datenbank mit vielfältigen Abfragemöglichkeiten zugänglich gemacht werden können.

Schrittweise auf ein Historisch syntaktisches Verbwörterbuch des Deutschen online (HSVW) hinzuarbeiten, ist das Anliegen einer seit 2012 agierenden internationalen Forschergruppe (vgl. Greule / Korhonen 2016, Vorwort). Das HSVW soll die Grundlage liefern, auf der Valenzgeschichten geschrieben werden können. Durch die Beschreibung der syntaktischen Umgebung eines Verbs im Verlauf der deutschen Sprachgeschichte wird ein Beitrag zum semantischen und syntaktischen Wandel dieses einen Verbs geleistet; ist ein großer Bestand an Verben derart erfasst, dann können Aussagen zum Sprachwandel 
sowohl der deutschen Sprache insgesamt als auch sprachübergreifend universell getroffen werden.

\section{Fallbeispiel nhd. zahlen, ahd. zalôn, mhd. zaln}

Wir wollen Péter Bassolas Beschreibung, wie die Satzbaupläne bzw. Valenzangaben in allgemeinen zweisprachigen Wörterbüchern geartet sind (Bassola 2006: 1388-1390), gleichsam umkehren und fragen, wie es damit in der deutschen sprachgeschichtlichen Lexikographie aussieht. Auch bei dem dazu gewählten Beispiel schließen wir uns Péter Bassola an und wählen das Verb zahlen. Methodisch gehen wir dabei so vor, als ob unser Ziel der Wortartikel mit dem Lemma zahlen für das geplante HSVW sei. Das bedeutet, dass wir uns nur auf Wörterbuch-Digitalisate stützen und am PC die in den Wortartikeln gespeicherten Valenzinformationen herausarbeiten, Satzbaupläne formulieren sowie die Ergebnisse in einem Word-Dokument speichern (vgl. Burghardt/ Reimann 2016). Dabei entdecken wir umgehend die Schwierigkeit, dass die Digitalisierung der Periodenwörterbücher noch nicht beim Buchstaben Z angekommen ist - mit Ausnahme des (1998-2018) voll digitalisierten Deutschen Wörterbuchs der Brüder Grimm. Auch das vom Institut für deutsche Sprache (IdS) erarbeitete Valenzwörterbuch deutscher Verben ist vollständig unter der Sigle e-valbu online verfügbar.

Ein Klick auf das Verb zahlen im e-valbu zeigt an, dass das Verb im Nhd. drei Bedeutungen hat und damit polysem ist:

zahlen1 ,jemand zahlt jemandem bzw. an jemanden etwas

zahlen2 ,jemand zahlt jemandem bzw. an jemanden für etwas irgendwieviel $^{c}$

zahlen3 ,jemand zahlt für etwas mit etwas‘.

Es ergibt sich daraus erstens die Erkenntnis, dass die Gesamtbedeutung des Verbs zahlen aus mehreren Sememen besteht, und zweitens die Frage, ob die Polysemie seit Anbeginn der Überlieferung des Verbs bestand, wie dann die Polysemie strukturiert war, wie viele Sememe beteiligt waren und wann Strukturveränderungen eintraten (Zum Semem vgl. Greule 2014: 57-59). Zur Be- 
schreibung des Verbsemems gehören die an der Verb-,„Szene“ beteiligten Rollen (Tiefenkasus, Argumente), die durch die Ergänzungen an der „Oberfläche“ markiert werden. Zur Szene von zahlen 1 gehören die Rollen Agens (der Zahlende), Adressat (der, dem gezahlt wird) und Objekt (das Gezahlte, Geldsumme). Daraus ergibt sich der Tiefenkasusrahmen (TKR) Agens-Adressat-Objekt (,Geldsumme'), das semantische Pendant zum morphologisch formulierten Satzbauplan $\mathrm{K}_{\text {sub }}, \mathrm{K}_{\mathrm{akk}},\left(\mathrm{K}_{\mathrm{dat}} / \mathrm{K}_{\mathrm{prp}}\right)$ im e-valbu [K= Konstituente].

\subsection{Sememe, Satzbaupläne und Tiefenkasusrahmen}

Das Valbu liefert zu zahlen1-3 zwar je einen Satzbauplan mit Belegungsregeln und Beispielsätzen, aber keinen Tiefenkasusrahmen (TKR). Wir schreiben die für zahlen1-3 im Valbu formulierten Satzbaupläne um (vgl. auch Bassola 2006: 1388) und setzen TKR und SBP zueinander in Beziehung:

zahlen1: Agens $\rightarrow$ NGnom // (Adressat $\rightarrow$ NGdat / PräpGan) // Objekt(Geldsumme) $\rightarrow$ NGakk

(lies: Agens wird ausgedrückt durch Nominalgruppe im Nominativ; Adressat fakultativ, ausgedrückt durch Nominalgruppe im Dativ oder Präpositionalgruppe mit an; Objekt ausgedrückt durch Nominalgruppe im Akkusativ)

Beispielsatz: Ein Mieter // muss // dem Vermieter // nicht in jedem Fall // Schadenersatz zahlen.

zahlen2: Agens $\rightarrow$ NGnom // (Adressat $\rightarrow$ NGdat / PräpGan) // Objekt ${ }^{1}$ Geldsumme) $\rightarrow$ NGakk // Objekt² (etwas, für das bezahlt wird) $\rightarrow$ PräpGfür

Beispielsatz: Herr Müller // muss // seiner Versicherung // viel Geld // für seine Alterssicherung // zahlen.

zahlen3: Agens $\rightarrow$ NGnom // Objekt $\rightarrow$ PräpGfür // Instrument $\rightarrow$ PräpGmit

Beispielsatz: Frau Schmidt // zahlte // für ihre Unaufmerksamkeit am Steuer // mit ihrem Leben. 
Um die Grundlage für die Valenzgeschichte von zahlen digital zu schaffen, kann man zurzeit nur auf den Artikel mit dem Lemma zahlen im Deutschen Wörterbuch der Brüder Grimm (DWB) online, Band 31, 1956, Sp. 44-56, zurückgreifen. Er ist nicht, wie es für die Darstellung der Entwicklung vorteilhaft wäre, nach Sprachperioden geordnet, sondern nach (ausführlich beschriebenen) Sememen, mit sporadischen valenzrelevanten Informationen, bietet aber eine große Zahl von aus den Quellentexten ausführlich zitierten Beispielen (zu Wörterbüchern als Quellen der historischen Valenz-Forschung vgl. Csiky / Greule 2008; Prinz 2016: 18-24).

Zusammengefasst und verkürzt unterscheidet das DWB zum Lemma zahlen historisch fünf Sememe:

(1) zusammen rechnen, zählen

(2) zurechnen, zu etwas zählen

(3) bezahlen

(4) durch Zahlung entschädigen, befriedigen

(5) erstatten, vergelten

Aus den im DWB den Sememen zugeordneten Beispielsätzen aus den historischen Quellen können folgende Satzbaupläne + Tiefenkasusrahmen rekonstruiert werden:

Ad (1) Semem ,zusammen rechnen, zählen' (schon im Ahd. um das Jahr 1000 belegt, DWB zitiert aber keinen ahd. Beispielsatz ${ }^{1}$ )

Agens (der Zählende) $\rightarrow$ NGnom //

Objekt (gezählte Elemente) $\rightarrow$ NGakk

Mhd. Beispielsatz: die bistume die dise wyhebischofe hant, die zalet man nüt, wan sü das mereteil ligent in der heidenschaft d. städtechron. 8, 405, 17. Noch im 16.Jh. belegt.

1 E. G. Graff, Althochdeutscher Sprachschatz, 5. Teil, 1840, Sp. 643, zitiert folgende ahd. Sätze: uuile du nu zálôn mit iro; (ih) zalon dir alliu miniu iar; (du) zálôst ube du zálôst; zalo mit mir. 
Ad (2) Semem ,zurechnen, zu etwas zählen

$$
\begin{aligned}
& \text { Agens (der Zählende) } \rightarrow \text { NGnom // } \\
& \text { Objekt (gezählte Elemente) } \rightarrow \text { NGakk // } \\
& \text { Ziel } \rightarrow \text { PräpG } z \text { ů }
\end{aligned}
$$

Mhd. Beispielsatz: die vorgenanten zwene, Otho und Vitellius, die zalt men nüt zu keysern, wan sü mit bosheit und falsche an das rich koment d. städtechron. 8, 344, 20.

\section{Ad (3) Semem ,bezahlen}

(3.1) Agens (der Zahlende) $\rightarrow$ NGnom

Beispiel 18.Jh. ich glaube nicht, dasz er zahlen kann J. GoтtнeLf schuldenb. 188.

(3.2) Agens (der Zahlende) $\rightarrow$ NGnom //

Objekt $^{2}$ (etwas, für das bezahlt wird) $\rightarrow$ PräpGan

Beispiel 18.Jh. der posten fand sich in der rechnung,

ich weisz, wir haben noch daran zu zahlen

SCHiller 12, 72 (Piccol. 1, 2).

\section{(3.3) Agens (der Zahlende) $\rightarrow$ NGnom // \\ Adressat (Mensch, dem man etwas schuldet) $\rightarrow$ NGdat}

Beispiel 18.Jh. ich zahle dir in einem andern leben,

gib deine jugend mir,

nichts kann ich dir als diese weisung geben

SCHILLER 4, 28 (resignation).

(3.4) Agens (der Zahlende) $\rightarrow$ NGnom //

Objekt ${ }^{1}$ (Geldsumme, die bezahlt wird) $\rightarrow$ NGakk 
Beispiel 16.Jh. als die mesz vollbracht ward, fürt sy der pfaff ins wirthshausz, zalt die ürten (Rechnung) Wickram rollw. 86, 6 Kurz;

(3.5) Agens (der Zahlende) ) $\rightarrow$ NGnom //

Objekt ${ }^{2}$ (etwas, für das bezahlt wird) $\rightarrow \mathrm{NGakk} / /$ Instrument(womit bezahlt wird) $\rightarrow$ PräpGmit

Beispiel 18.Jh. (er) zahlt heilig gaukelspiel mit seinem gut mit freuden HaLLER 149, 124 Hirzel.

Ad (4) Semem, jemanden durch Zahlung entschädigen, befriedigen

(4.1) Agens (der Zahlende/Entschädigende) $\rightarrow$ NGnom // Adressat (Mensch, dem man etwas schuldet) $\rightarrow$ NGakk

Beispiel 16.Jh. der meister dir geliehen hat, das du solt zalen den gwandschneyder H. SACHs fastn. sp. 1, 41, 145 Götze.

(4.2) Agens (der Zahlende/Entschädigende) $\rightarrow$ NGnom // Adressat (Mensch, dem man etwas schuldet) $\rightarrow$ NGakk // Instrument (womit bezahlt wird) $\rightarrow$ PräpGmit

Beispiel 17.Jh. wer schuld mit schulden zahlt, thut selten alles gut, der letzte, der jhm borgt, den zahlt er mit dem hut (d. i. als bettelmann)

LogAU 3, 27, 23.

\section{(Ad 5) Semem, erstatten, vergelten}

(5.1) Agens (Vergeltender) $\rightarrow$ NGnom // Adressat (dem vergolten wird) $\rightarrow$ NGdat // Objekt (was vergolten wird) $\rightarrow \mathrm{NGakk}$ 
Beispiel 18.Jh. er soll mir zahlen die todesblässe auf diesen wangen! soll mir zahlen die vernichtung, die an diesem herzen saugt! soll mir zahlen die starren thränen, die an diesen augen hängen KLINGER 2, 28.

(5.2) Agens (Erstattender) $\rightarrow$ NGnom //

Objekt (was erstattet wird) $\rightarrow \mathrm{NGakk} / /$

Instrument(womit erstattet wird) $\rightarrow$ PräpGmit

Beispiel: (sie werden) mit unsrer armuth ihre länderkäufe, mit unserm blute ihre kriege zahlen SCHILLER 14, 312 (Tell 2, 1).

\section{Valenzgeschichte zum Verb zahlen}

Die Valenzgeschichte nimmt ihren Ausgang bei der Etymologie des Lemma-Verbs: Ahd. zalôn ist vom Substantiv ahd. zala ,Zahl, Anzahl, Reihe, Menge abgeleitet wie ahtôn ,achten' von ahd. ahta ,Gedanke, Betrachtung. Das DWB formuliert die Grundbedeutung so: „rechnerisch ausführen, nach den regeln der zahlenkunst vor- oder darlegen: ahd. zalôn, calculum ponere, considerare numerum, numeros replicare, reputare; mhd. besonders gesagt von der beschäftigung auf der mittelalterlichen rechenmaschine, dem zahlbret, daher dann allgemein wie zusammen rechnen, zählen. "Damit wird Semem 1 die zweiwertige Aktantenkonstellation [Agens (der Zählende) $\rightarrow$ NGnom // Objekt (gezählte Elemente) $\rightarrow$ NGakk] zugeschrieben. Abgesehen davon, dass Semem1 unter dem Aspekt, dass etwas zu etwas rechnerisch hinzugefügt wird, durch die Aufnahme der Rolle Ziel zu Semem2 spezifiziert wurde, unterliegt Semem1 einem tief greifenden Bedeutungswandel: Er kann so erklärt werden, dass die Szene des Zählens spezifiziert wurde und auf das Zählen von Geld und die Übergabe der gezählten Summe an einen „Empfänger“ (= bezahlen) fokussiert wurde. ${ }^{2}$ Der

\footnotetext{
2 Im DWB wird der Bedeutungswandel folgendermaßen erklärt: „die spätere und heutige bedeutung der erlegung einer summe, die zahlen hat, geht von der angeführten mittelalterlichen verwendung des zahlbrets aus, welches bei allen gröszeren berechnungen in anwendung kam, und vom gebrauche der rechenpfennige [...] von anfang an ist dabei das verbum als bezahlen häufiger im gebrauch als in einfacher form."
} 
Bedeutungswandel macht sich in einer Ausweitung auf mehrere Subsememe mit teils dreiwertiger Aktantenkonstellation bemerkbar. Der Hintergrund ist der, dass an der Szene des Bezahlens mehr als drei Rollen beteiligt sein können: Agens (Zahlender), Adressat / Empfänger, Zahlungsmittel / Instrument, Objekt ${ }^{1}$ (Geldsumme, die bezahlt wird) und Objekt ${ }^{2}$ (etwas, für das bezahlt wird); sie sind aber nie gemeinsam als Ergänzungen in einem Satz realisiert.

Während in Semem4 die Szene erfasst wird, dass ein Geschädigter durch Zahlung einer Summe entschädigt wird und der geschädigte ,Empfänger' wider Erwarten mit einem Satzglied im Akkusativ bezeichnet wird, wird zahlen im Semem5, veranlasst durch Dichter des 18.Jh., metaphorisch ohne Bezug auf Geldsummen im Sinne von ,erstatten, vergelten, zurückgeben' als dreiwertige Aktantenkonstellation, in der entweder der Adressat oder das Instrument genannt werden, realisiert.

Vergleicht man die aus dem DWB rekonstruierten Valenzinformationen unter dem Lemma zahlen mit den Angaben des E-Valbu, so wird deutlich, dass sich zum Deutsch der Gegenwart hin das Semem3 durchgesetzt und alle anderen in der Sprachgeschichte auftauchenden Sememe verdrängt hat. Die ursprüngliche Polysemie ist auf das monoseme Verb nhd. zahlen reduziert worden.

Die (3-wertige) Aktantenkonstellation von zahlen1 [Agens $\rightarrow$ NGnom // (Adressat $\rightarrow$ NGdat / PräpGan) // Objekt(Geldsumme) $\rightarrow$ NGakk] ist zwar im DWB nicht belegt, stellt aber eine Kombination der historischen Subsememe 3.3 [Agens (der Zahlende) $\rightarrow$ NGnom // Adressat (Mensch, dem man etwas schuldet) $\rightarrow$ NGdat] und 3.4 [Agens (der Zahlende) $\rightarrow$ NGnom // Objekt ${ }^{1}$ (Geldsumme, die bezahlt wird) $\rightarrow$ NGakk] dar.

Ebenso ist die (4-wertige) Aktantenkonstellation von zahlen2 [Agens $\rightarrow$ NGnom // (Adressat $\rightarrow$ NGdat / PräpGan) // Objekt ${ }^{1}$ (Geldsumme) $\rightarrow$ NGakk // Objekt ${ }^{2}$ (etwas, für das bezahlt wird) $\rightarrow$ PräpGfür] eine Kombination der historischen Subsememe 3.2 [Agens (der Zahlende) $\rightarrow$ NGnom // Objekt ${ }^{2}$ (etwas, für das bezahlt wird) $\rightarrow$ PräpGan], 3.3 [Agens (der Zahlende) $\rightarrow$ NGnom // Adressat (Mensch, dem man etwas schuldet) $\rightarrow$ NGdat] und 3.4 [Agens (der Zahlende) $\rightarrow$ NGnom // Objekt $^{1}$ (Geldsumme, die bezahlt wird) $\rightarrow$ NGakk]. Lediglich tritt im Nhd. bei der Rolle Objekt ${ }^{2}$ (etwas, für das bezahlt wird) anstelle der PräpGan die PräpGfür.

Zahlen3 steht zu zahlen1-2 in metaphorischem Verhältnis: In dieser Szene wird nicht mit Geld, sondern mit ideellen Werten „bezahlt“. Zahlen3 steht damit dem historischen Semem5 nahe und die nhd. (3-wertige) Aktantenkonstellati- 
on [Agens $\rightarrow$ NGnom // Objekt $\rightarrow$ PräpGfür // Instrument $\rightarrow$ PräpGmit] entspricht dem historischen Subsemem 5.2 [Agens $\rightarrow$ NGnom // Objekt (was erstattet wird) $\rightarrow$ NGakk // Instrument(womit erstattet wird) $\rightarrow$ PräpGmit], wobei bei der Rolle Objekt an die Stelle der Kasus-Ergänzung im Nhd. die PräpGfür tritt.

\section{Valenzgeschichte zum Verb bieten}

Wenn wir die in Kapitel 2 oben entworfene Valenzgeschichte von zahlen mit der unten folgenden Valenzgeschichte von bieten vergleichen, dann zeigt sich, dass die Geschichte von bieten auf einer weit größeren Materialbasis und viel differenzierter entworfen werden konnte. Die Gründe dafür liegen nicht nur bei der Tatsache, dass sich bieten nahezu konkurrenzlos im Nhd. als Verb etablieren konnte, sondern vor allem darin, dass aus den digitalisierten Sprachstadienwörterbüchern, die aber noch nicht bei $\mathrm{Z}$ angelangt sind, wesentlich mehr Informationen zur Syntax der Verben erschlossen werden können, als in dem zwar ebenfalls digitalisierten, aber nicht nach Sprachstadien geordneten DWB der 1. Auflage.

Aus ahd. biotan, mhd., frnhd., nhd. bieten wird im Vergleich mit got. -biudan, awn. bióða, ae. bēodan, afr. biada, as. biodan ein urgermanisches starkes Verb der 2. Ablautreihe ${ }^{\star} b e u d-a$ - erschlossen. Obwohl ${ }^{\star} b e u d-a$ - problemlos auf idg. ${ }^{*} b^{h}$ éud ${ }^{h}-e$ - (Präsens) ,wach werden, aufmerksam werden' zurückgeführt werden kann, stellt die Bedeutung ,bieten' des germanischen Verbs demgegenüber eine Neuerung dar, die sich aus dem Bedeutungskomplex ,zur Aufmerksamkeit veranlassen, kundtun, gebieten, darbieten' entwickelt haben soll (Pfeifer 1989: 169).

Im Verlauf der deutschen Sprachgeschichte fällt das Verb bieten durch eine Bedeutungsvielfalt sowohl in den einzelnen historischen Sprachperioden als auch in der gesamten Sprachgeschichte auf. Zeitweise wird das Verb sogar als Funktionsverb (Bildung von Streckformen) grammatikalisiert (DWB Neubearbeitung, S. 209f.; Frnhd.Wb. bieten Bed.2; auch Mhd.Wb. bieten 5.Phras.)

Die Bedeutungsentfaltung lässt sich auf eine Grundbedeutung zurückführen, in der verschiedene Aspekte einer "Szene" hypothetisch zusammengefasst sind und aus der heraus sich die einzelnen Sememe (frames) als Perspektivierung und Fokussierung eines Teilaspekts erklären lassen (vgl. Welke 2011: 154f.). Wir gehen davon aus, dass die (germanische) Ursprungsbedeutung von 
bieten einen Aspekt der bekannten Kaufszene (oder Tauschszene) abbildete, nämlich dass ein Mensch x ein Objekt y einem Menschen z zum Kauf oder Tausch anbietet. Es handelt sich dabei gleichsam um die Eröffnungsphase des ganzen Tausch- oder Kaufvorgangs, die durch ein „dreiwertiges“ Verbsemem mit dem Kasusrahmen oder den Rollen Agens („Anbietender“) - Objekt („Angebotenes") - Adressat versprachlicht ist.

Der Ursprungsbedeutung kommt die im DWB (Neubearbeitung 2013, Sp. 206 213) als „hauptgebrauch“ an die Spitze gestellte Bedeutung am nächsten:

\section{Semem A}

,jmdm. etw. anbieten, übergeben (wollen); jmdm. etwas in Aussicht, zur Verfügung stellen, gewähren (wollen).

Das Semem A ist in allen Wörterbüchern verzeichnet und wird wie folgt beschrieben: ,etw. anbieten, darreichen; das Angebotene ist etw. Reales und soll in den Besitz oder die Nutzung des andern übergehen: Speise und Trank; ein Entgelt; ein Dankopfer; ein Ersatz; der Kaufpreis oder das Lösegeld in einem Han-

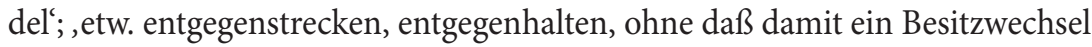
eintritt; ein Glied, einen Körperteil (zu einem besonderen Zweck) darbieten; etw. als Lockmittel vorhalten, darbieten' (Ahd.Wb.); ,jmdm. etw. anbieten', ,(jmdm.) etw. (Speise, Trank usw.) darreichen, geben' (Mhd.Wb.); '(jmd.) etw. darbieten, anbieten, geben' (Frnhd.Wb.); ,(jmdm.) etw. (als Gegenwert) anbieten, in Aussicht stellen; zeigen, vor Augen stellen; (jdm), etw. reichen, hinhalten (mehrf. im Bild); , hin-, zu-, darreichen; geben, gewähren, darbringen' (Änhd./ Goethe-Wb.); ,jemand stellt jemandem etwas zur Verfügung; zur Nutzung anbieten', jemand gewährt jemandem etwas', jemand zeigt jemandem jemanden/ etwas; darbieten' (nhd./Valbu).

Kasusrahmen und Satzbaupläne:

Ahd. TKR: Agens (Mensch) - Adressat (Mensch) - Objekt (real) SBP: NGnom - NGdat - NGakk

Mhd. TKR: Agens (Mensch) - Adressat (Mensch) - Objekt (konkret) SBP: NGnom - NGdat - NGakk 
Frnhd. TKR: Agens (Mensch/Gott) - (Adressat) - Objekt (konkret)

SBP: NGnom - (NGdat) - NGakk

Änhd. TKR: Agens (Mensch) - Adressat (Mensch) - Objekt

SBP: NGnom (Mensch) - (NGdat) - NGakk

Nhd. TKR(a): [Agens - Objekt (konkret) - (Adressat)]

SBP(a): NGnom - NGakk - (NGdat)

TKR(b): [Agens - Objekt - Adressat]

SBP(b): NGnom - NGakk - NGdat

TKR (c): [Agens - (Objekt) - Adressat]

SBP(c): NGnom - NGakk - (NGdat).

Das Semem B kommt durch Spezifikation (Bedeutungsverengung) der Grundbedeutung zustande, indem das Angebotene auf den Verkauf fokussiert ist und der Verkaufspreis als eine Kasusrolle WERT hinzukommt. Es ist - außer im Ahd. - in allen Sprachperioden belegt und wird im DWB (Neubearbeitung 2013, Sp. 206f.) in zwei Subsememen (1.c und 1.d) ausformuliert. Durch die Aufnahme einer weiteren Rolle (Wert) wird die Grundvalenz vierwertig (vgl. das frnhd. Semem B), wird aber fast immer nur dreiwertig unter Aussparung der Adressaten-Rolle gebraucht.

,zum Verkauf anbieten, einen (bestimmten) Kaufpreis verlangen, ,eine Summe als Kaufpreis, Lösegeld u. dgl. nennen; Gebot (bei einer Auktion) abgeben

Beschreibungen in den Wörterbüchern: ,etw. zum Kauf anbieten', ,auf etw. bieten' (eine Geldsumme auf/für etwas bieten)` (Mhd.Wb.); etw. zum Verkauf anbieten, verkaufen' (Frnhd.Wb.); ,im Handel u Zahlungsverkehr: eine Ware anbieten', ,etw zum Verkauf bieten'; , für eine Ware, Immobilien u.a. ein Kaufgebot machen; bei einer Auktion ein Gebot machen, in einer Wette dagegensetzen; als Ausdruck der Bereitschaft, die Wette einzugehen' (Änhd., Goethe-Wb.); ,jemand bietet jemandem irgendwieviel für etwas' (Valbu).

Kasusrahmen und Satzbaupläne:

Mhd. TKR(a): Agens (Mensch) - Objekt - Wert/Grad 
SBP(a): NGnom - NGakk - Adv/PräpGumb

TKR(b): Agens (Mensch) - Objekt (das Angebotene, Geldsumme) - Ziel

SBP(b): NGnom - (Adv) - (PräpGûf ).

Frnhd. TKR: Agens (Mensch) - Objekt - (Adressat, Mensch) - Wert (Geldsumme)

SBP: NGnom - NGakk - (NGdat) - PräpGvur/umbe/Adv/Adj

Änhd. TKR(a): Agens (Mensch) - Objekt - Wert (Preis, Geldsumme)

SBP(a): NGnom - NGakk - PräpGzu

TKR(b): Agens (Mensch) - Objekt - Wert

SBP(b): NGnom - PräpGauf - PräpGbis/NGakk

Nhd. TKR: [Agens - Adressat- Objekt (affiziert) - Menge]

SBP: NGnom - (NGdat) - PräpGfür+akk/ADVdafür - NGakk.

Bei Semem C wechselt die Perspektive vor dem Hintergrund der Ausgangsbedeutung von bieten vom Zahlenwert auf den immateriellen (ideellen) Wert des Angebotenen, das sich in einer Haltung des Anbieters äußert. Außer im Nhd. wird das Semem C in allen deutschen Sprachperioden versprachlicht. Das DWB (Neubearbeitung 2013, Sp. 209f.) formuliert dazu zwei Subsememe (3.a und 3.c)

,jmdm. oder etw. ein bestimmtes Verhalten entgegenbringen; Huld, Ehre erweisen, in einer bestimmten Weise mit jmdm. umgehen, ihn behandeln; jmdn. (zu etwas) herausfordern, gegen jmdn. oder etwas Widerstand leisten

Beschreibungen in den Wörterbüchern: ,erzeigen, erweisen; das Angebotene äußert sich in einer menschlichen Haltung' (Ahd.Wb.); ,in einer bestimmten Weise mit jmdm. umgehen, ihn behandeln` (Mhd.Wb.); ,(jmd.) etw. entgegenbringen, erweisen, entbieten ' (Fnhd.Wb.); ,(einen Gruß) entbieten, Ehre erwei-

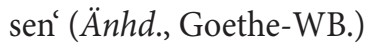


Kasusrahmen und Satzbaupläne:

Ahd. TKR: Agens (Mensch) - Adressat (Mensch) - Patiens

SBP: NGnom - NGdat - NGakk

Mhd. TKR: Agens (Mensch) - Objekt (affiziert, Mensch) - Modus SBP: NGnom - NGdat - Adv

Frnhd. TKR: Agens (Mensch) - Adressat (Mensch/Gott) - Patiens SBP: NGnom - NGdat - NGakk

Änhd. TKR: Agens (Mensch) - Adressat - Inhalt (positiv)

SBP: NGnom - NGdat - NGakk

Der Fokus kann auch auf dem Aspekt liegen, dass der Darbietende in der Kauf-/Tauschszene mit verschiedenen Zwecksetzungen sich sprachlich äußert, was semanto-syntaktisch zur Folge hat, dass zur Grundvalenz die Kasusrolle INHALT (des Geäußerten) hinzukommt. Bis auf die spezifizierende Weiterführung zum Semem E ,befehlen, gebieten' ist dieser Frame nur in einzelnen Sememen unterschiedlicher Sprachperioden und aufgrund von Erfordernissen in den Fachsprachen, vor allem im Ahd., vorhanden. Zusammen mit Semem E gehört Semem D zum Wortfeld der Mitteilung (Sprachproduktion) (Sommerfeld / Schreiber 1996: 144-146).

Semem D1: ,etw. zur Kenntnis bringen, bekannt machen; verkündigen, lehren Ahd. TKR: Agens (Mensch) - Inhalt (Worte, Begriffe)

SBP: NGnom - NGakk

Semem D2: ,zum Vergleich darbieten, als Vergleich setzen;,etw. zum Vergleich darbieten

Ahd. TKR: Agens (Mensch) - Inhalt - zu Vergleichendes

SBP: NGnom - NGakk - PräpG ze/zuo

Mhd. TKR: Agens - zu Vergleichendes - Inhalt

SBP: NGnom - PräpGze - NGakk 
Semem D3: ,voraussetzen, als Voraussetzung (beim Schließen) vorbringen Ahd. TKR: Agens (Mensch) - Inhalt SBP: NGnom - NGakk

Semem D4: ,jmdn. um Vermittlung bitten, jmds. Entscheidung fordern, sich auf jmdn. berufen

Frnhd. TKR: Agens (Mensch) - Adressat (Mensch) - Inhalt (Sachen)

SBP(a): NGnom - NGakk - NGgen

SBP(b): NGnom - PräpGuff/von - NGakk.

Bei Semem E, das seit dem Mhd. in den Texten dreier Sprachperioden vertreten ist (und in der gotischen Bibel mit dem Präfixverb anabiudan wiedergegeben wird), wird der Frame von Semem D dadurch spezifiziert und verengt, dass der Inhalt der Rede von einem Agens, das jemandem gebieten/befehlen kann, geäußert wird. Das DWB (Neubearbeitung 2013, Sp. 210f.) formuliert dazu drei Teilsememe (4, 4.a und 4.b)

jmdm. etwas befehlen, gebieten oft rechtlich; jmdn. an einen bestimmten Ort beordern, etwas anordnen, bestimmen; jmdm. etwas vorschreiben

Beschreibungen in den Wörterbüchern: ,jmdm. etw. gebieten, befehlen' (Mhd. Wb.); ,(jmdm.) etw. empfehlen, befehlen, gebieten, vorschreiben' (Frnhd.Wb.); ,gebieten, Anweisungen geben' (nur in Götz u Werth II Ossian) (Änhd., Goethe-Wb.). (Im Nhd. wird die Bedeutung von Semem E vom Präfixverb gebieten übernommen und dadurch verdrängt.)

Kasusrahmen und Satzbaupläne:

Mhd. TKR: Agens (Mensch) - Adressat (Mensch) - Inhalt

SBP: NGnom - NGdat - NSdaz

Frnhd. TKR: Agens - (Adressat, Mensch) - Inhalt

SBP: NGnom - (NGdat) - IK/NSdas 
Änhd. TKR: Agens (Mensch) - Adressat (Mensch) - Inhalt SBP: NGnom - NGdat - NS.

Aus der Verkaufsszene kann schließlich abgeleitet werden, dass der Anbietende das angebotene Objekt zum Käufer hin bewegt. Auf diese Weise erklärt sich das im Ahd. und Mhd. belegte Semem F, in dessen Kasusrahmen ein Ort bzw. ein Ziel, zu dem hin das Objekt bewegt wird, aufgenommen wird. Erfasst werden damit:

- $\quad$ das ahd. Semem 5 ,etw. zu oder vor jmdn. bringen', mit

TKR: Agens (Mensch) - Zielort - Patiens

SBP: NGnom - PräpGbifora - NGakk;

- $\quad$ das mhd. Semem 4 ,etw. hinstrecken, hinhalten

TKR: Agens (belebt) - (Adressat) - Objekt (konkret) - Ziel

SBP: NGnom - NGdat - NGakk - Adv/PräpG vür/gein

- $\quad$ das mhd. Semem 5 ,etw. wohin führen, bewegen', mit

TKR: Agens (Mensch) - Objekt - Zielort

SBP: NGnom - NGakk - PräpG ze/gein/Adv, sowie

- $\quad$ das änhd. Semem 3 ,jn., etw. in eine bestimmte Richtung lenken, an einen bestimmten Ort schicken; sich an einen bestimmten Ort begeben', mit TKR: Agens (Mensch) - Objekt - Zielort/Richtung SBP: NGnom - NGakk/dat - PräpGzu/gên/vor/in/Adv.

Vereinzelt und ohne direkte diachrone Vergleichsmöglichkeit sind:

- $\quad$ das änhd. Semem 2: ,(jdm) etw als Gegenleistung, Belohnung, Entschädigung uä versprechen, zusichern', mit

TKR: Agens (Mensch) - (Adressat) - Objekt - Gegenleistung

SBP: NGnom - (NGdat) - NGakk - PräpGfür ;

- $\quad$ das nhd. Semem 3 ,jemand/etwas stellt jemandem bzw. für jemanden etwas dar', mit

TKR: [Agens - Objekt - Adressat]

SBP: NGnom - NGakk - (NGdat/PräpGfür+akk), und

- $\quad$ das nhd. Semem 7 ,jemand/etwas hat etwas als Erscheinungsbild' [geh], mit TKR: [Agens - Objekt (effiziert)]

SBP: NGnom - NGakk. 


\section{Zusammenfassung und Ausblick}

Wir sind eingangs Péter Bassola gefolgt und haben Satzbaupläne und Tiefenkasusrahmen als wichtigste Informationen zu Verben in einem Wörterbuch festgestellt. Es wurde dann beobachtet, dass die historische Valenzlexikographie, deren Aufgabe es wäre, die historische Entwicklung der Satzbauplan-Tiefenkasusrahmen-Konstellation festzuhalten, nach ersten Anläufen zum Stillstand kam. Ursachen dafür waren das Problem der Quellen und vor allem die Tatsache, dass sowohl die historischen Quellen als auch die historischen Wörterbücher digitalisiert wurden und werden. Da inzwischen auch das Valenzwörterbuch (Valbu) elektronisch vorliegt, liegt es auf der Hand erstens an die Ausarbeitung eines Historisch syntaktischen Verbwörterbuch zu gehen und zweitens dafür die Digitalisate der Sprachstadienwörterbücher (ahd., mhd., fnhd., änhd.) und des DWB (1. Auflage) zu nutzen und gewissermaßen auf dieser Grundlage einerseits die Menge der Satzbaupläne / Tiefenkasusrahmen direkt am PC zu rekonstruieren und abzuspeichern sowie andererseits aus diesen Informationen eine Geschichte der Entwicklung der syntaktisch-semantischen Umgebung (Valenzgeschichte) für jedes Verb zu schreiben. Schließlich bekunden auch Vertreter der Konstruktionsgrammatik Interesse an dem geplanten HSVW (vgl. Rostila 2016), um es unter dem Aspekt ihres neuen Forschungsansatzes auszuwerten.

Der Vergleich der Valenzgeschichte von zahlen, die noch nur auf der Grundlage des digitalisierten DWB (1. Auflage) verfasst wurde, mit der Valenzgeschichte von bieten zeigt, um wieviel größer und genauer die Valenzinformationen sind, wenn sich die Forschung auf die in den historischen Wörterbüchern gespeicherten Informationen stützen kann. Eine wichtige Voraussetzung für das Gelingen des Forschungsprojekts kann hier nur angedeutet werden. Es geht darum, die Menge der Verben, die in der gesamten deutschen Sprachgeschichte seit dem Ahd. belegt sind und in das HSVW aufgenommen werden sollten, festzustellen. Ferner muss die Frage geklärt werden, wie das Verhältnis der Bearbeitung der Simplicia zur Bearbeitung der mit Präfix derivierten Verben (z.B. ahd. geltan, firgeltan, intgeltan, bezahlen', nhd. gelten, vergelten, entgelten) aussehen und erfolgen soll. 


\section{Literatur}

Ágel, Vilmos et al. (Hrsg.) (2006): Dependenz und Valenz. Ein internationales Handbuch der zeitgenössischen Forschung. Bd. 2., Berlin / New York: de Gruyter (HSK 25.2).

Bassola, Péter (2006): Valenzinformationen in allgemeinen zweisprachigen Wörterbüchern. In: Ágel, Vilmos et al. (Hrsg.) (2006): Dependenz und Valenz. Ein internationales Handbuch der zeitgenössischen Forschung. Bd. 2., Berlin / New York: de Gruyter (HSK 25.2), 1387-1396.

Burghardt, Manuel /Reimann, Sandra (2016): Möglichkeiten der elektronischen Aufbereitung und Nutzung eines historisch syntaktischen Verbwörterbuchs des Deutschen. In: Greule, Albrecht / Korhonen, Jarmo (Hrsg.), 300-322. Csiky, Nandor (2008): Das Wortfeld WACHSEN im Deutschen. Studien zu seiner Struktur in Gegenwart und Geschichte. Hamburg: Dr. Kovač.

Csiky, Nandor / Albrecht Greule (2008): Wörterbücher als Quellen der historischen Valenz-Forschung am Beispiel des Verbs verzeihen. In: Lefèvre, Michel / Simmler, Franz (Hrsg.): Historische Syntax und Semantik vom Althochdeutschen bis zum Neuhochdeutschen. Festschrift für Yvon Desportes zum 60. Geburtstag. Berlin: Weidler, 371-377.

Greule, Albrecht (2014): Diachrone Perspektiven im Historischen deutschen Valenzwörterbuch. In: Glottotheory 5.2, 53-63.

Greule, Albrecht (2018): Das Mittelhochdeutsche Wörterbuch online und die Valenz. Aus der Werkstatt des Mittelhochdeutschen syntaktischen Verbwörterbuchs. In: Czajkowski, Luise / Ulbrich-Bösch, Sabrina / Waldvogel, Christina (Hrsg.): Sprachwandel im Deutschen. Berlin/Boston: de Gruyter (Lingua Historica Germanica 19), 67-75.

Greule, Albrecht / Korhonen, Jarmo (Hrsg.) (2016): Historisch syntaktisches Verbwörterbuch. Valenz- und konstruktionsgrammatische Beiträge. Frankfurt a.M., Bern, Bruxelles: Lang (Finnische Beiträge zur Germanistik 34). Pfeifer, Wolfgang (1989): Etymologisches Wörterbuch des Deutschen, 3 Bände, erarbeitet von einem Autorenkollektiv des Zentralinstituts für Sprachwissenschaft unter der Leitung von Wolfgang Pfeifer, Berlin: Akademie-Verlag. Prinz, Michael (2016): Wörterbücher und digitale Belegrepositorien als Quellen für ein historisch syntaktisches Verbwörterbuch. Am Beispiel des benefaktiven Dativs bei mhd. bachen. In: Greule, Albrecht / Korhonen, Jarmo (Hrsg.), 17-53. 
Rostila, Jouni (2016): Zur Integration von Argumentstrukturkonstruktionen in das Historisch syntaktische Verbwörterbuch. In: Greule, Albrecht / Korhonen, Jarmo (Hrsg.), 261-276.

Schmid, Hans Ulrich (2017): Einführung in die deutsche Sprachgeschichte, 3., überarb. und aktual. Aufl. Stuttgart: Metzler.

Sommerfeld, Karl-Ernst / Schreiber, Herbert (1996): Wörterbuch der Valenz etymologisch verwandter Wörter. Verben, Adjektive, Substantive, Tübingen: Niemeyer.

Welke, Klaus (2011): Valenzgrammatik des Deutschen. Eine Einführung. Berlin, New York: de Gruyter.

\section{Internet-Quellen}

Althochdeutsches Wörterbuch online: http://awb.saw-leipzig.de (gesichtet am 12.08.2018).

DWB. Deutsches Wörterbuch der Gebrüder Grimm, online-Version: http:// dwb.uni-trier.de (gesichtet am 12.08.2018).

E-VALBU: Das elektronische Valenzwörterbuch deutscher Verben: https:// grammis.ids-mannheim.de/verbvalenz (gesichtet am 12.08.2018).

Frühneuhochdeutsches Wörterbuch: FWB-online: https://fwb-online.de (gesichtet am 12.08.2018).

Goethe-Wörterbuch online: http://gwb.uni-trier.de/de (gesichtet am 12.08. 2018).

Mittelhochdeutsches Wörterbuch: http://www.mhdwb-online.de (gesichtet am 12.08.2018). 